Revue des patrimoines

$2 \mid 2002$

La monographie d'architecture

\title{
La collégiale de Saint-Bonnet-le-Château (Loire)
}

\section{Bernard Ducouret}

\section{(2) OpenEdition}

Journals

Édition électronique

URL : http://journals.openedition.org/insitu/1176

DOI : 10.4000/insitu. 1176

ISSN : 1630-7305

\section{Éditeur}

Ministère de la culture

\section{Référence électronique}

Bernard Ducouret, «La collégiale de Saint-Bonnet-le-Château (Loire) », In Situ [En ligne], 2 | 2002, mis en ligne le 23 avril 2012, consulté le 20 avril 2019. URL : http://journals.openedition.org/insitu/1176 ; DOI : 10.4000/insitu. 1176

Ce document a été généré automatiquement le 20 avril 2019.

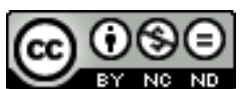

In Situ Revues des patrimoines est mis à disposition selon les termes de la licence Creative Commons Attribution - Pas d'Utilisation Commerciale - Pas de Modification 4.0 International. 


\title{
La collégiale de Saint-Bonnet-le- Château (Loire)
}

\author{
Bernard Ducouret
}

La collégiale de Saint-Bonnet-le-Château est principalement connue pour les peintures murales du XV siècle qui décorent sa chapelle basse, dont une partie est reproduite au musée des Monuments français, et, à un moindre degré, pour sa bibliothèque qui conserve de nombreux incunables. Son architecture, plus ignorée, n'en est pas moins digne d'intérêt. Nous ne présenterons pas ici l'ensemble de l'édifice, négligeant des éléments remarquables comme le porche sud (cf. plan, au-dessous de la sacristie 5) ou la chapelle Sainte-Cécile-et-Saint-Benoît (actuellement Saint-Eloi, cf. plan, $n^{\circ} 7$ ), mais nous nous attacherons à mettre en valeur quelques problèmes de chronologie (fig. $\left.\mathbf{n}^{\circ} \mathbf{1}\right)$ et de lecture de l'œuvre qui se sont posés lors de la réalisation de la monographie, faite dans le cadre de l'inventaire du canton de Saint-Bonnet-le-Château. Cette approche fragmentaire nécessite auparavant une présentation générale de l'édifice.

\section{Présentation de l'édifice}




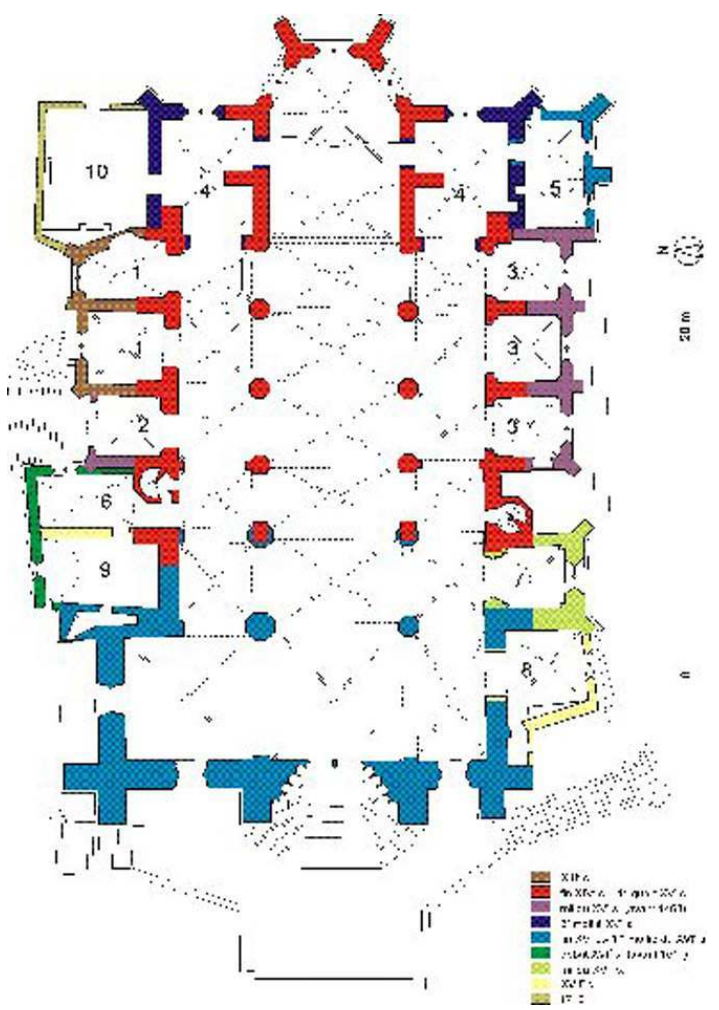

Plan chronologique

Dess. Inv. P. Cherblanc d'après un relevé par J.-F. Grange-Chavanis en 1994. (c) Inventaire général, ADAGP, 1994

2 Saint-Bonnet-le-Château est une petite ville sise dans les monts du Forez, à $850 \mathrm{~m}$ d'altitude, au sud-ouest du département de la Loire, à proximité du Puy-de-Dôme et de la Haute-Loire. Elle appartenait au diocèse de Lyon avant la création, récente, de celui de Saint-Etienne, mais se trouvait dans une position extrême, à la limite de ceux de Clermont-Ferrand et du Puy. Aussi est-elle peu éloignée de l'abbaye de La Chaise-Dieu dans le rayonnement de laquelle se place l'architecture de son église. La ville est construite sur une éminence dont la collégiale occupe le point le plus élevé, immédiatement à côté du site de l'ancien château (fig. $n^{\circ} 2$ ). 


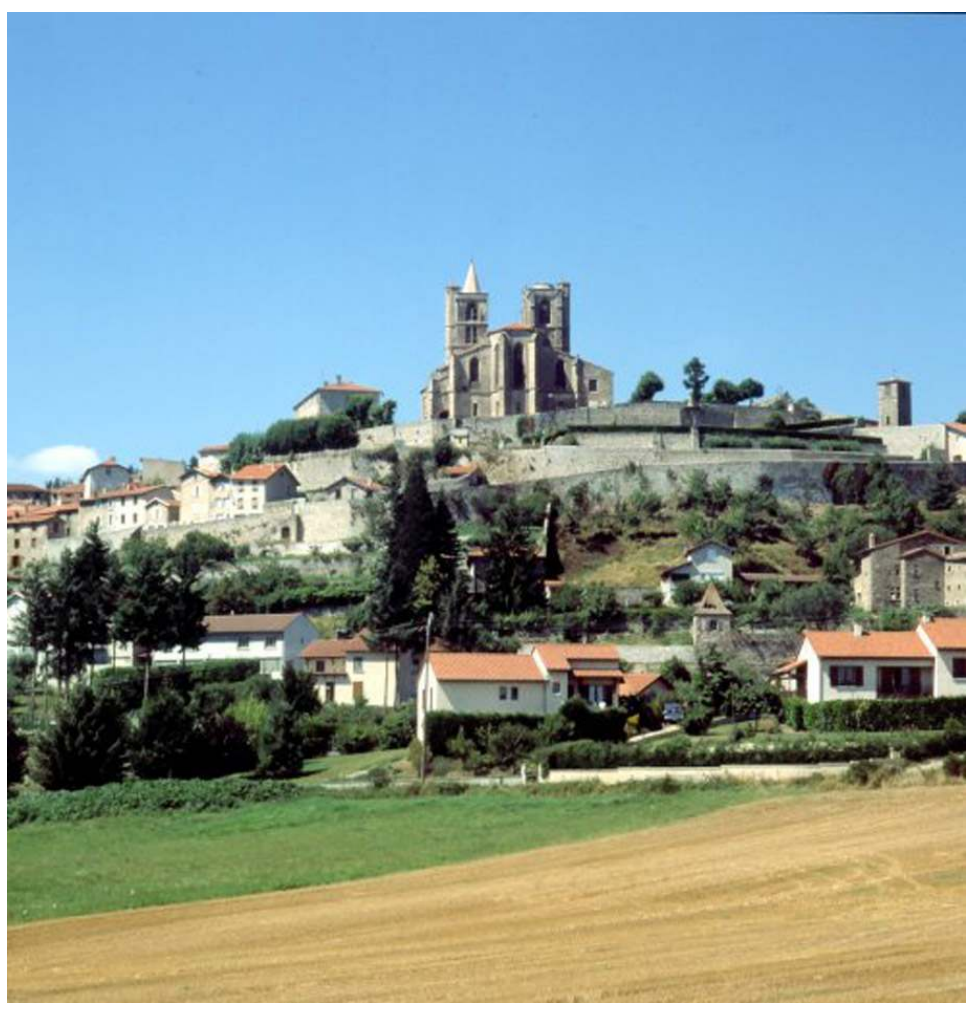

La collégiale dans son site, côté chevet

Phot. Inv. J.-M. Refflé. @ Inventaire général, ADAGP, 1996

Comme l'indique son nom, Saint-Bonnet-le-Château est un bourg castral qui connaît son apogée aux $\mathrm{XIV}^{\mathrm{e}}$ et $\mathrm{XV}^{\mathrm{e}}$ siècles. A cette époque, c'est avec Saint-Galmier la ville marchande la plus active du Forez sur le nouveau chemin reliant Lyon à Toulouse ${ }^{1}$. Son industrie du fer est réputée : entre 1344 et 1350, ses forgerons fournissent sur le chantier de La Chaise-Dieu des barres ou des plaques de fer qui sont retravaillées sur place ${ }^{2}$.

Le lieu de culte est mentionné pour la première fois en 1225 dans un pouillé du diocèse de Lyon, sous l'appellation de capella relevant de la paroisse de Saint-Nizier-de-Fornas, dont le centre est à quelques kilomètres de là̉ . Cette église est érigée en paroisse à une date inconnue que Condamin et Langlois situent entre 1351 et 1361. En effet, à cette dernière date apparaissent le curé, Mathieu Bolle, et une communauté de prêtres sociétaires, tandis qu'en 1351 Mathieu Bolle est cité seulement comme «clerc juré en la cour du Forez $»^{4}$.

Les communautés de prêtres sociétaires ou prêtres desserviteurs, dont le rôle principal est d'assurer les fondations de messes pour les défunts, sont nombreuses dans le Forez à la fin du Moyen Age. Mais celle de Saint-Bonnet est tout à fait à part dès l'origine. Contrairement aux autres, elle est régie par des ordonnances qui, en 1520, sont renouvelées et promulguées sous forme de statuts. C'est ainsi que le groupe, sous la direction du curé, s'assimile à un véritable chapitre de chanoines : plusieurs membres ont une fonction déterminée, tous portent un habit particulier qui les distingue; dans les textes se rencontrent les expressions $\mathrm{d}^{\prime}$ " office canonial» et de «congrégation collégiale ». Il possède un quartier, dit « le cloître ", qui semble correspondre à une partie de l'îlot situé à l'ouest de l'église et où se trouvent le presbytère avec son jardin et son 
verger montant jusqu'à l'église, plusieurs logements pour les prêtres et une grande salle dite le "Cénacle » ${ }^{5}$. L'église même abrite un « cabinet des archives », mentionné en 1760 et $1793^{6}$, et une bibliothèque installée dans un corps spécial édifié en 1716-17177.

6 Les prêtres forment l'épicentre d'un foyer culturel important dans la province. Ils fondent dès le XIV $\mathrm{X}^{\mathrm{e}}$ siècle une école qui acquiert rapidement un rayonnement régional d'une centaine de kilomètres à la ronde, mais c'est principalement la qualité de leur pratique musicale qui les singularise et les rend célèbres dans le diocèse $\mathrm{du} \mathrm{XV}^{\mathrm{e}}$ au XVIII ${ }^{\mathrm{e}}$ siècle ${ }^{8}$.

7 Le nouveau statut de l'église de Saint-Bonnet à la fin du XIV ${ }^{e}$ siècle impose vite la nécessité de construire un édifice de plus vastes dimensions. Les travaux s'échelonnent tout au long $\mathrm{du} \mathrm{XV} \mathrm{XV}^{\mathrm{e}}$ et du XVI $\mathrm{XI}^{\mathrm{e}}$ siècle, lui conférant une silhouette assez surprenante, principalement à cause des deux tours qui surgissent du centre de la nef. Cependant, le bâtiment étant élevé tout en pierre de taille, en granite, il est aisé d'en lire toutes les coutures, d'en définir les adjonctions les unes par rapport aux autres et de retrouver le programme d'origine ${ }^{9}$. La figure 1 donne le résultat de cette investigation dont nous allons analyser plus en détail certains aspects.

\section{La chapelle primitive}

Figure 3

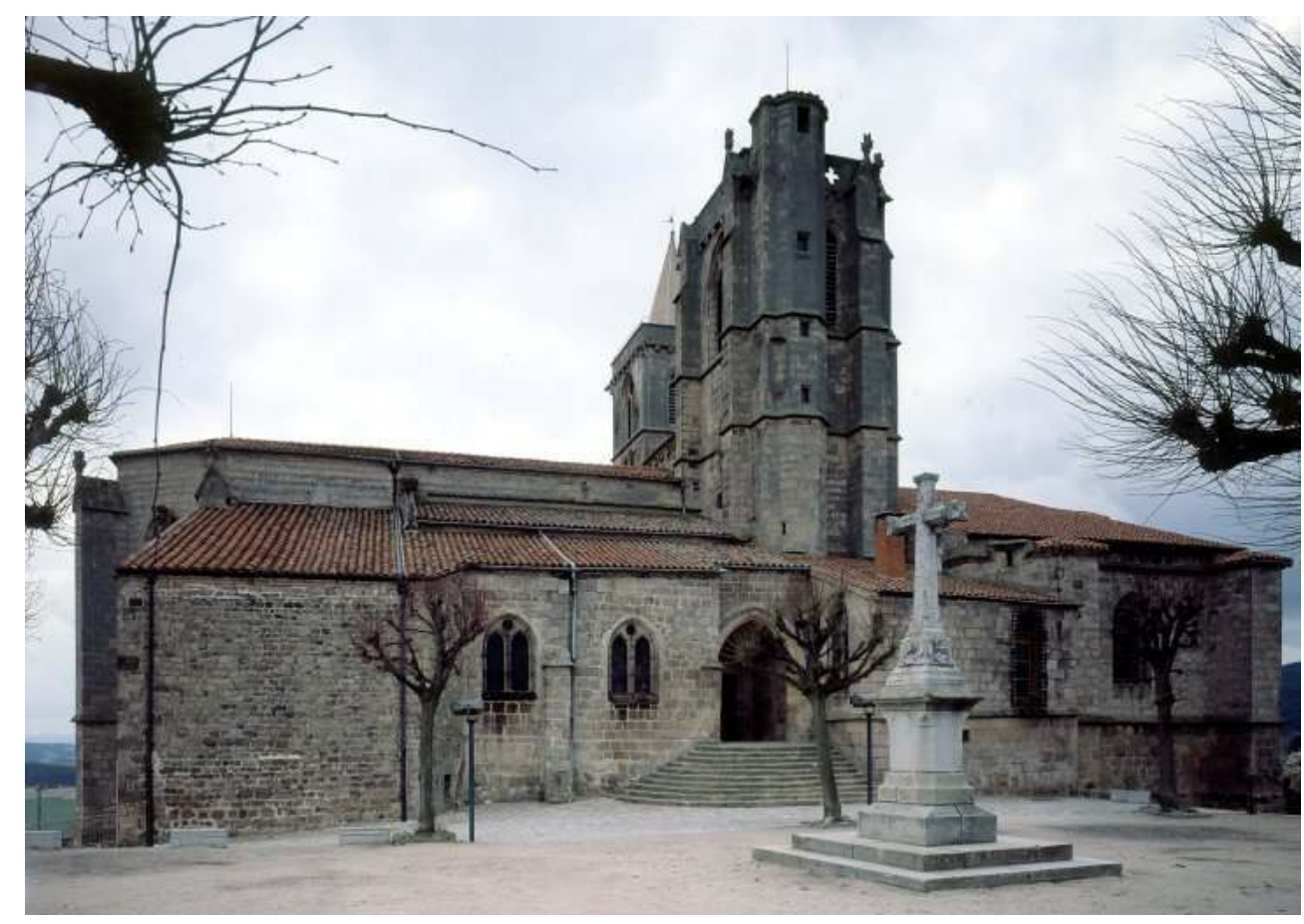

Vue générale du côté nord

Phot. Inv. J.-M. Refflé. (C) Inventaire général, ADAGP, 1998

8 Les parties les plus anciennes sont les deux chapelles marquées 1 sur le plan. On remarque que l'une dessine un curieux biais à l'est que rien a priori ne justifie. Sur l'élévation extérieure, les baies à deux formes surmontées d'un oculus sont caractéristiques du XIII ${ }^{\mathrm{e}}$ siècle (fig. $\mathrm{n}^{\circ} 3$ ). 
Figure 4

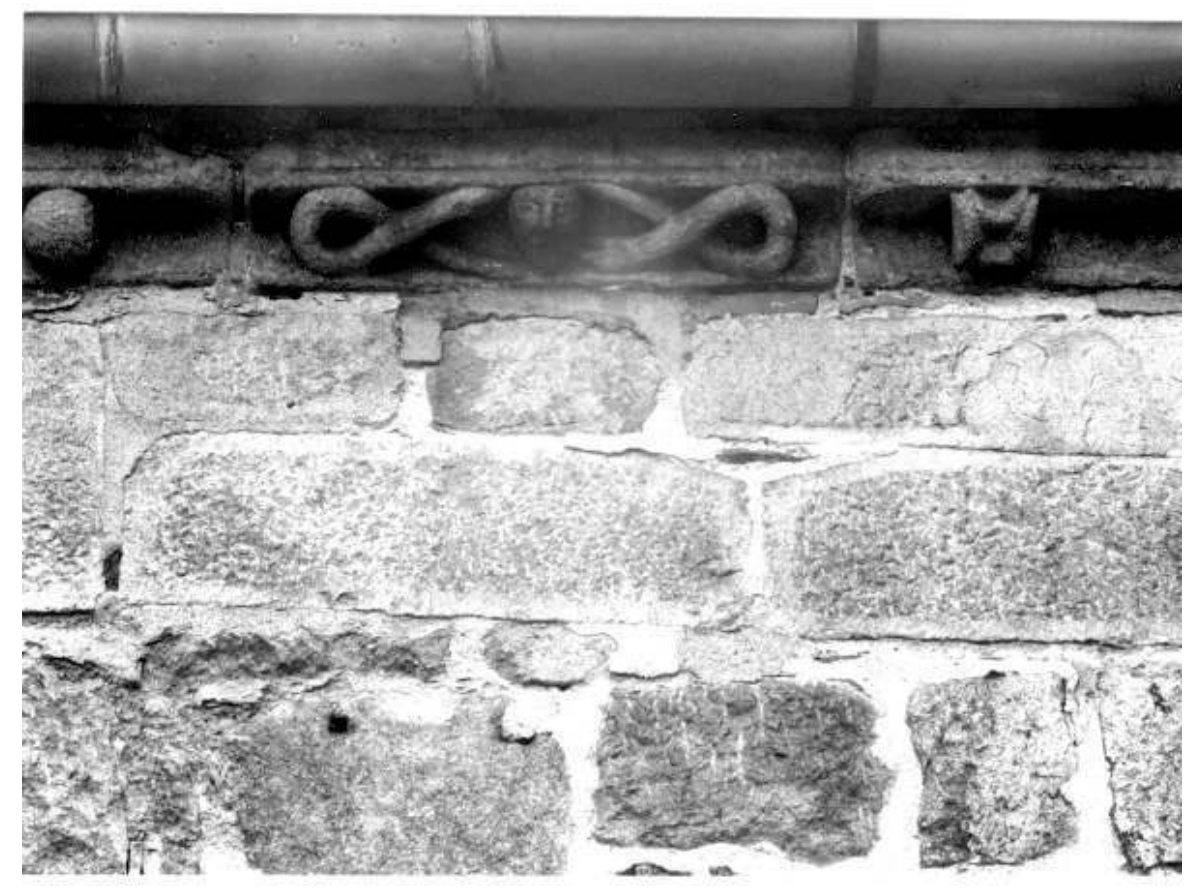

Chapelles 1 (vestiges de l'église primitive), détail de la corniche

Phot. Inv. J.-M. Refflé. (c) Inventaire général, ADAGP, 1998

9 En revanche, la corniche à cavet (fig. $\left.n^{\circ} 4\right)$, sur laquelle se détachent des motifs sculptés, s'apparente à des modèles du XII ${ }^{e}$ siècle présents dans cette partie du Forez, dont l'église du prieuré de Rozier-Côtes-d'Aurec est l'exemple le plus accompli ${ }^{10}$. 
Figure 5

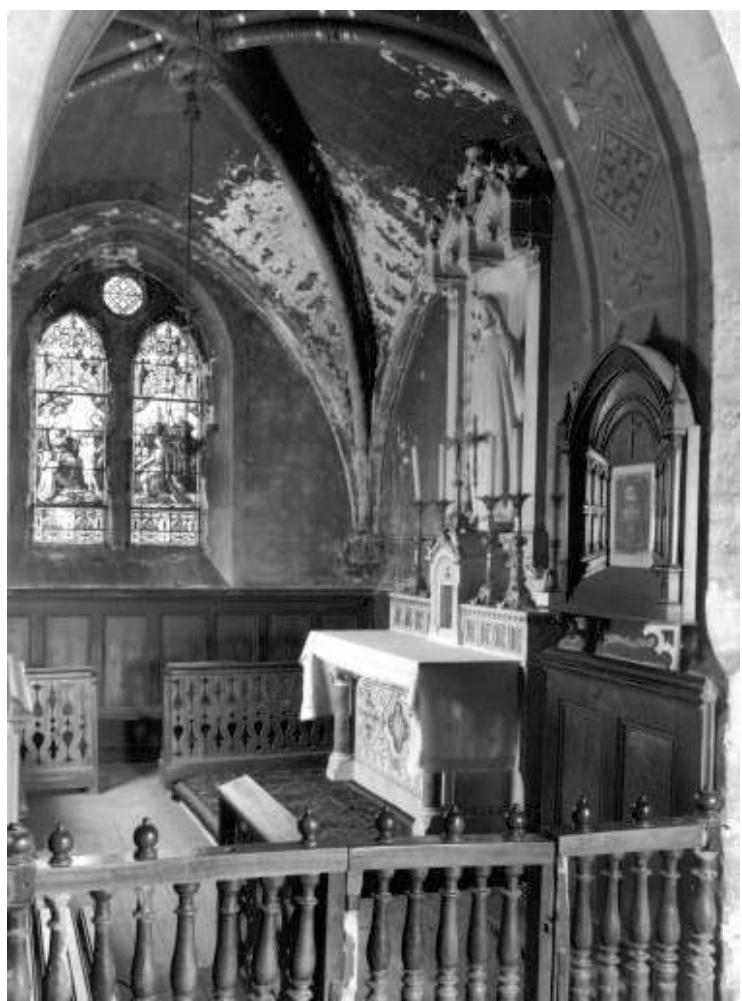

Chapelles 1 (vestiges de l'église primitive), vue intérieure de la première côté ouest Phot. Inv. J.-M. Refflé. () Inventaire général, ADAGP, 1998

10 A l'intérieur (fig. $\mathrm{n}^{\circ}$ 5), la voûte d'ogives à gros tore de la première chapelle est également du XIII ${ }^{\mathrm{e}}$ siècle, de même que les culots qui reçoivent les nervures. Cependant, l'incohérence de ces derniers par rapport au mur témoigne d'une reprise postérieure. Ces éléments constituent donc des vestiges de la première église, partiellement remontés pour former deux chapelles latérales à la nouvelle. La structure date visiblement du XIII siècle, mais on y a rapporté une moulure du XII ${ }^{e}$ siècle, la corniche, provenant probablement d'une partie détruite de l'édifice. La corniche donne également la preuve que l'église existait dès le XII ${ }^{e}$ siècle, c'est-à-dire antérieurement à la première mention de 1225.

\section{La nouvelle église et sa datation}


Figure 6

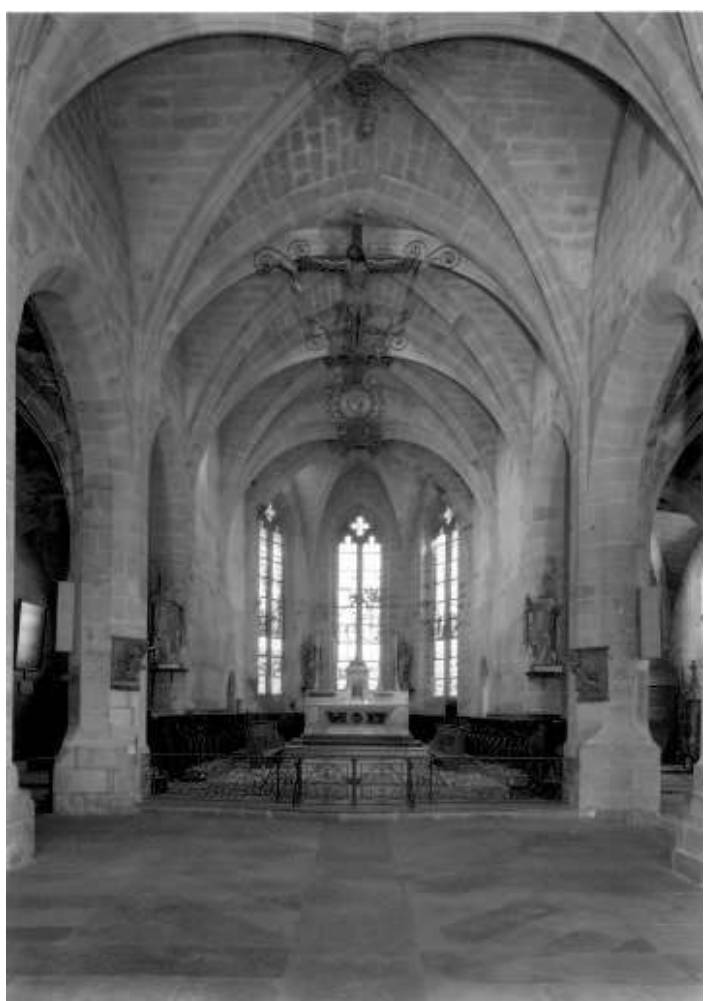

Vue intérieure des deux dernières travées de la nef et du chœur

Phot. Inv. E. Dessert, J.-M. Refflé. (c) Inventaire général, ADAGP, 1998

11 Dégagée de toutes les adjonctions postérieures, la nouvelle église apparaît en rouge sur le plan. Elle se distingue également par le fait que presque toutes les pierres portent des marques de tâcheron totalement absentes des agrandissements ultérieurs. Elle se compose d'une nef de quatre travées à trois vaisseaux, celui du centre étant légèrement plus haut que les collatéraux, d'un chœur simple à abside à trois pans (fig. $\mathrm{n}^{\circ} 6$ ). De grandes fenêtres dont les traces sont encore visibles éclairaient primitivement les deux travées droites de celui-ci. La déclivité du terrain s'accentuant vers l'est, un étage de soubassement qui contient une chapelle, est aménagé sous le chœur. Deux tours reliées par une terrasse s'élèvent au-dessus de la première travée de la nef. Le tout est très influencé par l'église abbatiale de La Chaise-Dieu (1344-1355) : absence de transept, trois vaisseaux à peu près d'égale hauteur, colonnes polygonales recevant directement les nervures sans l'intermédiaire de chapiteaux, hautes fenêtres de l'abside. 


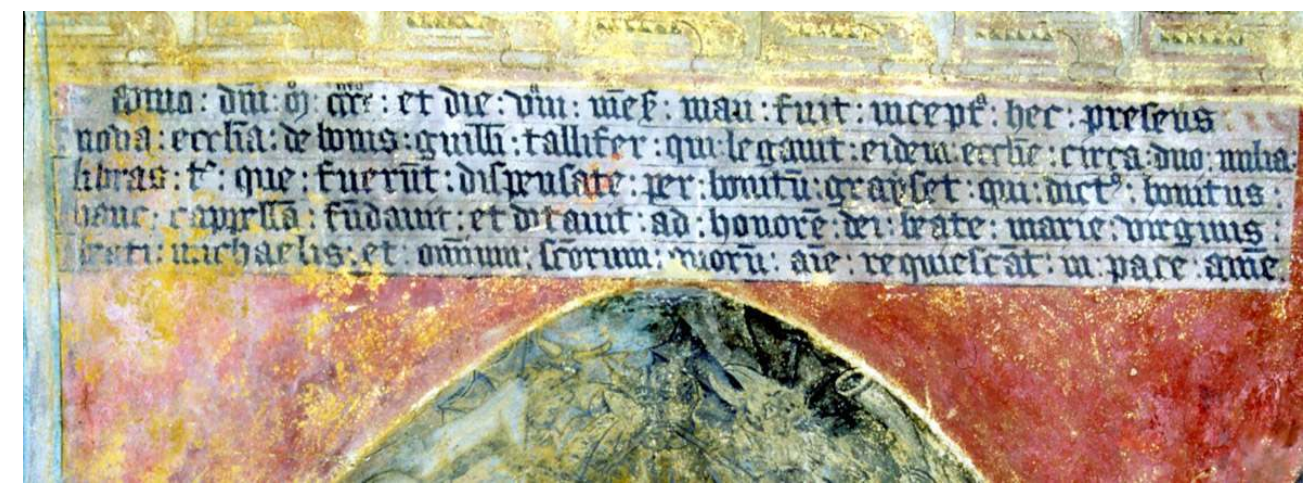

Chapelle basse sous le chœur, détail de l'inscription au-dessus de la porte nord

Phot. Inv. A. Franchella. () Inventaire général, ADAGP, 1996

La date du début des travaux est apparemment donnée par une inscription peinte sur le mur nord de la chapelle basse (fig. $n^{\circ} 7$ ), indiquant que le nouvel édifice est commencé le 8 mai 1400 grâce au legs de deux mille livres tournois par Guillaume Taillefer, qui a été dispensé par Bonnet Grayset. Notons que l'inscription se termine par cette formule: QUORUM : ANIMAE : REQUIESCANT : IN : PACE : AMEN, «que leurs âmes reposent en paix ». La marque du pluriel signifie qu'à cette date Bonnet Grayset lui-même est mort. Or nous savons, par l'épitaphe conservée de son tombeau, qu'il est décédé le 8 juin 1426, ce qui signifie que l'inscription a été peinte au moins vingt-six ans après la date présumée du début des travaux ${ }^{11}$.

D'autre part, dans son testament du 18 juin 1399, le même Guillaume Taillefer, marchand drapier, dit avoir entrepris « dès maintenant» l'édification du chœur pour les travaux duquel il lègue une somme d'argent: Item dat et legat affectione quam habet erga et in edificio chori ecclesie sancti Boniti, jam per ipsum incepto, operi et edificio ipsius chori et ecclesie... ${ }^{12}$. Vingt-sept ans plus tôt, en 1372, on trouve deux dons faits à l' « œuvre » de l'église " operi ecclesie Sancti Boniti $»^{13}$. Aussi, comme le suggèrent Condamin et Langlois, ces dons semblent signifier que dès 1372 la communauté se préoccupe de collecter l'argent nécessaire pour une reconstruction. Le chœur est déjà en travaux en juin 1399 et le 8 mai 1400 peut être considéré comme la date de la cérémonie officielle de début des travaux, sans doute celle de la pose de la première pierre. 


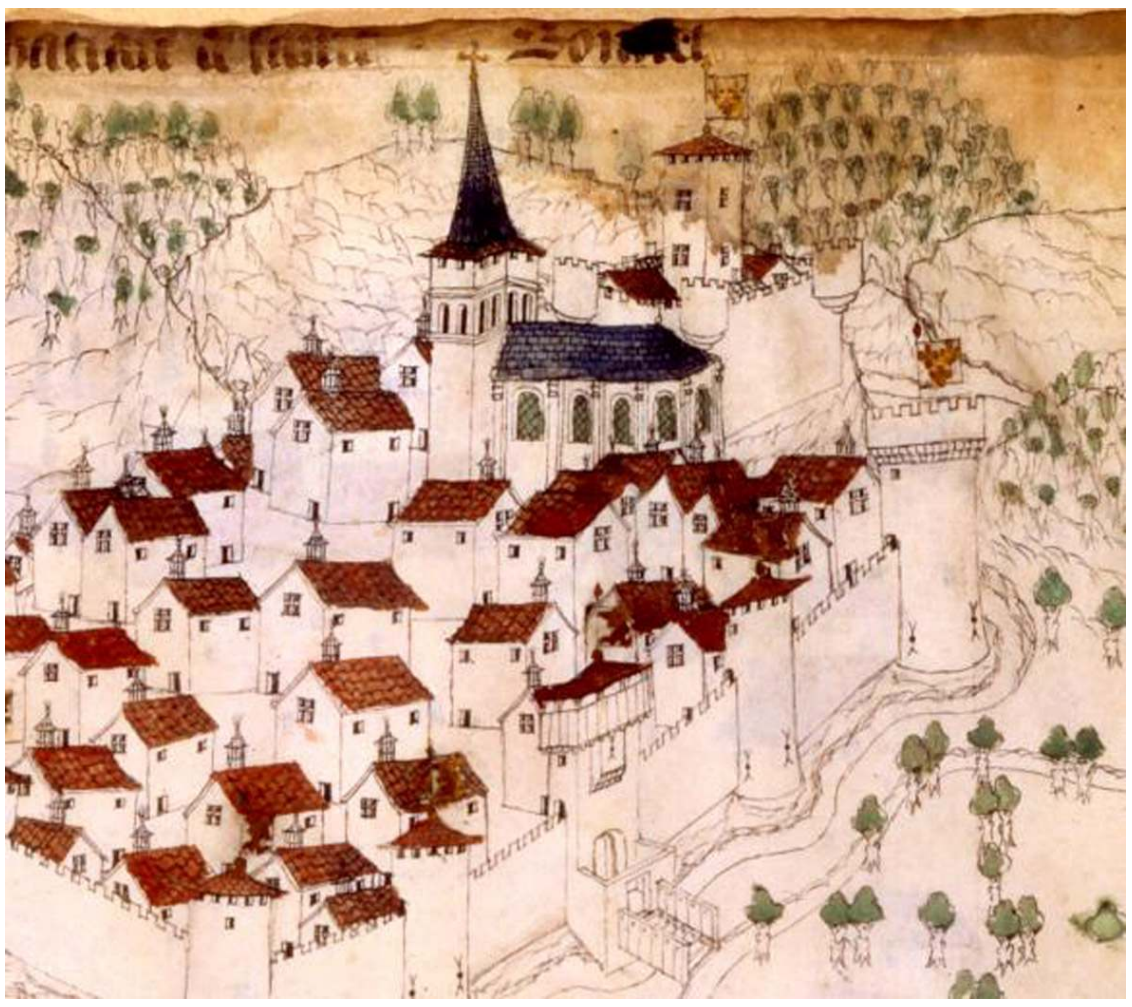

"La ville et chatiau de Saint-Bonnet », dessin extrait de : Guillaume Revel, Armorial du Forez, [ca 1450] (BN, fr. 2896, 2897, f. 471)

Phot. BN. (c) Bibliothèque nationale de France

La période d'achèvement du chantier est restée vague jusqu'à la découverte, par Fournial, du testament d'Etienne Brun, marchand originaire de Salers, rédigé en $1418^{14}$. Par ce document, il lègue une somme pour la fabrication d'une cloche (ad opus et ad faciendi unum simbalum in ecclesia $S B C$ predicti) et 60 sous tournois pour financer les vantaux d'un nouveau portail (dat et legat fabrice ecclesie $S B C$ qui fiet nova balva sive portali novum seaxaginta solidos $t$. semel) ${ }^{15}$. Ces legs permettent de supposer que le portail ouest et au moins un clocher sont achevés, autrement dit que les travaux de construction touchent à leur fin. L'église apparaît complètement terminée avec ses deux tours sur la vue de Guillaume Revel, dessinée vers 1450 (fig. $\mathrm{n}^{\circ}$ 8).

\section{Les tours}




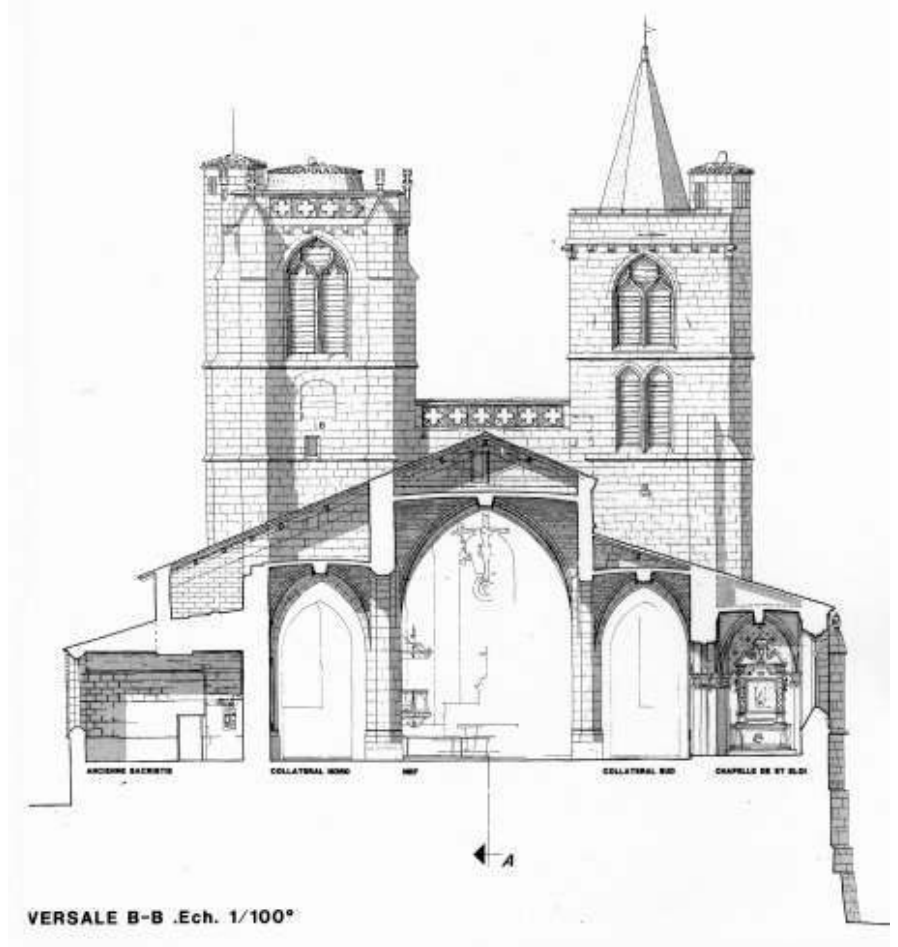

Coupe transversale sur la deuxième travée de la nef, par J.-F. Grange-Chavanis, 1994

Phot. Inv. E. Dessert. ( ) Inventaire général, ADAGP, 1999

Les deux tours encadrant la façade primitive (fig. $n^{\circ} 9$ ) présentent quelques différences : la tour nord, plus haute que celle du sud, est contrebutée sur toute sa hauteur par des contreforts, lesquels ne dépassent pas le niveau de la souche sur la tour sud; la première a un seul niveau de baies alors que la seconde en montre deux. Des éléments communs leur donnent malgré tout une unité : escalier montant de fond jusqu'à la coursière à la base de la flèche couronnant chaque tour à l'origine, cordons de séparation d'étage régnant à la même hauteur. A l'intérieur, la tour sud possède un rez-de-chaussée voûté, correspondant à la première travée du collatéral. Au-dessus, un étage peu éclairé est couvert par un plancher. Les deux niveaux de baies enferment un seul étage occupé par un grand beffroi qui soutenait onze cloches avant la Révolution ${ }^{16}$. Le rez-de-chaussée de la tour nord forme, comme son pendant, la première travée du collatéral. Cependant une dissemblance est marquée dans la voûte: alors qu'ici il s'agit d'une croisée d'ogives simple, au sud un oculus est ménagé pour le passage des cloches. Effectivement, ce système est inutile au nord puisque le premier étage carré de la tour nord est également voûté d'ogives. Le deuxième est couvert d'un berceau en arc segmentaire et possède une cheminée d'angle (fig. $n^{\circ} 10$ ). L'étage au-dessus, couvert par un plancher, communique avec la terrasse reliant les deux tours. Enfin l'étage ajouré contient les deux grosses cloches de l'église. Il en était probablement ainsi dès l'origine, car d'une part le prix-fait du bourdon actuel, passé en 1683, précise que la nouvelle cloche doit être de même dimension que l'ancienne et, d'autre part, une fondation de messe en 1506 exige que l'on fasse tinter l'une des grosses cloches du clocher Saint-André, vocable de la chapelle jouxtant la tour nord ${ }^{17}$. 
Figure 10

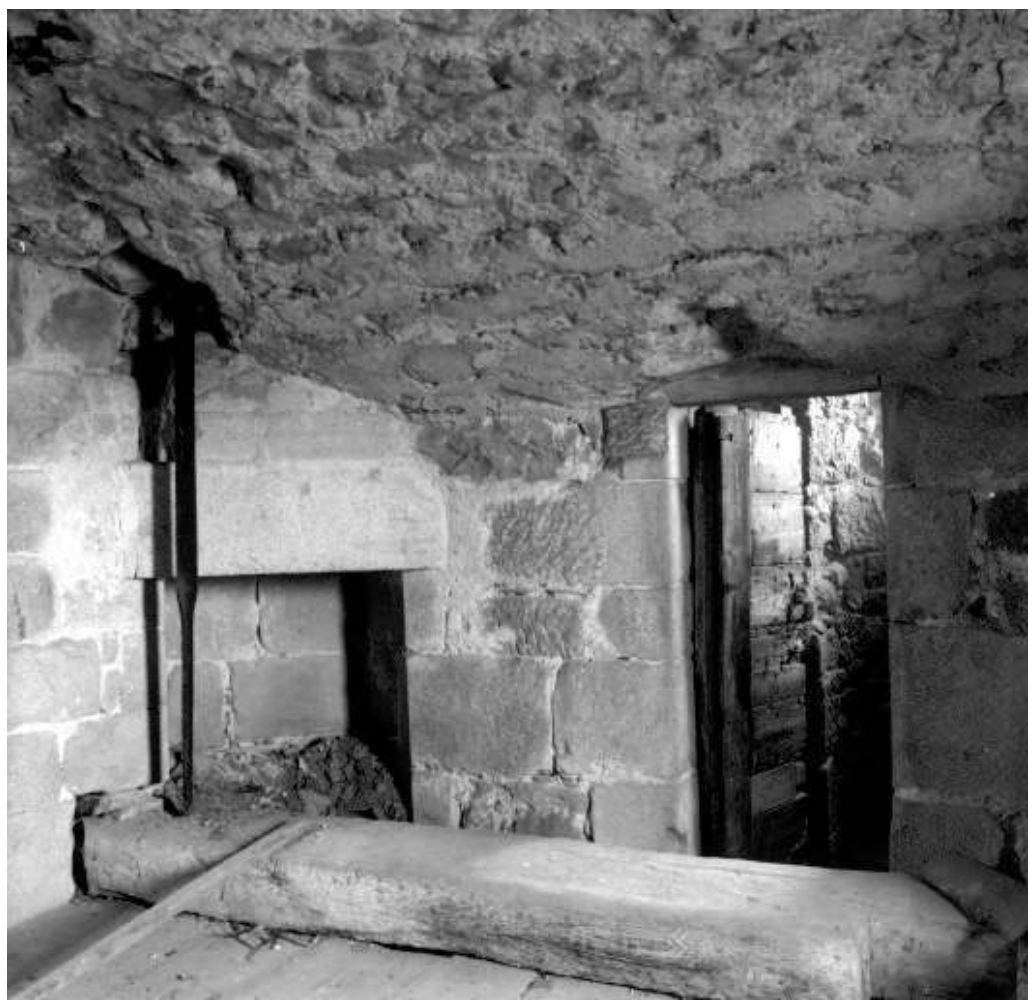

Tour nord, pièce du deuxième étage : cheminée et porte donnant sur l'escalier Phot. Inv. E. Dessert, J.-M. Refflé. (C) Inventaire général, ADAGP, 199

La structure interne explique donc certaines différences extérieures: d'un côté la présence de trois pièces voûtées superposées nécessite un contrebutement plus important des murs, non utile de l'autre côté où seul le rez-de-chaussée est voûté. Ces dissemblances traduisent également des fonctions distinctes. La tour sud est conçue principalement pour la sonnerie. La tour nord au contraire a plusieurs usages : le premier étage, soigneusement voûté, remplissait un rôle que nous ne connaissons plus : est-ce le cabinet des archives mentionné à plusieurs reprises ? ${ }^{18}$ La plus grande hauteur de cette tour, sur le point le plus élevé de la ville, lui assigne un rôle de guet indéniable, d'autant qu'elle est édifiée en pleine guerre de Cent Ans, époque où les fortifications tant de la ville que du château sont renforcées ${ }^{19}$. La coursière au sommet de la tour sud permet le même usage, d'autant que la circulation entre les deux tours est facilitée par la terrasse. Enfin elle sert également de clocher. L'étage à cheminée constitue plus vraisemblablement l'ancien logement du sonneur qu'une salle pour les gardes ${ }^{20}$.

Un autre problème d'interprétation des tours est lié aux flèches. Elles sont élevées en brique plombifère noire ${ }^{21}$. Avec le temps, il a fallu les recouvrir d'un enduit d'étanchéité. Le 9 mai 1627, la foudre frappe la flèche nord. Un chroniqueur contemporain rapporte que «l'esguille du dit clocher, presque consumée par le feu, tomba sur le couvert de la chapelle de M. Conchon » $\left(n^{\circ} 6 \text { et } 9 \text { sur le plan }\right)^{22}$. Il ajoute que le beffroi n'a pratiquement pas été touché et, effectivement, celui-ci porte encore la date de 1607. Seule la cloche de l'horloge a dû être refaite ${ }^{23}$. Se pose alors la question de savoir ce qui a bien pu brûler pour entraîner la chute d'une flèche en brique? Une réponse est donnée par la vue de Revel qui a dessiné à la base des flèches des appentis mettant à l'abri les coursières. Le 
noir de la figuration des flèches se distingue du rouge de la tuile qui protège les appentis. C'est donc vraisemblablement la structure en bois de ces appentis, à laquelle était peutêtre combiné le support de la cloche de l'horloge, qui a pris feu. Ces protections, fréquentes au-dessus des chemins de ronde des enceintes, confirment par ailleurs le rôle de guet de ces coursières.

\section{L'agrandissement de la nef vers l'ouest}

Figure 11

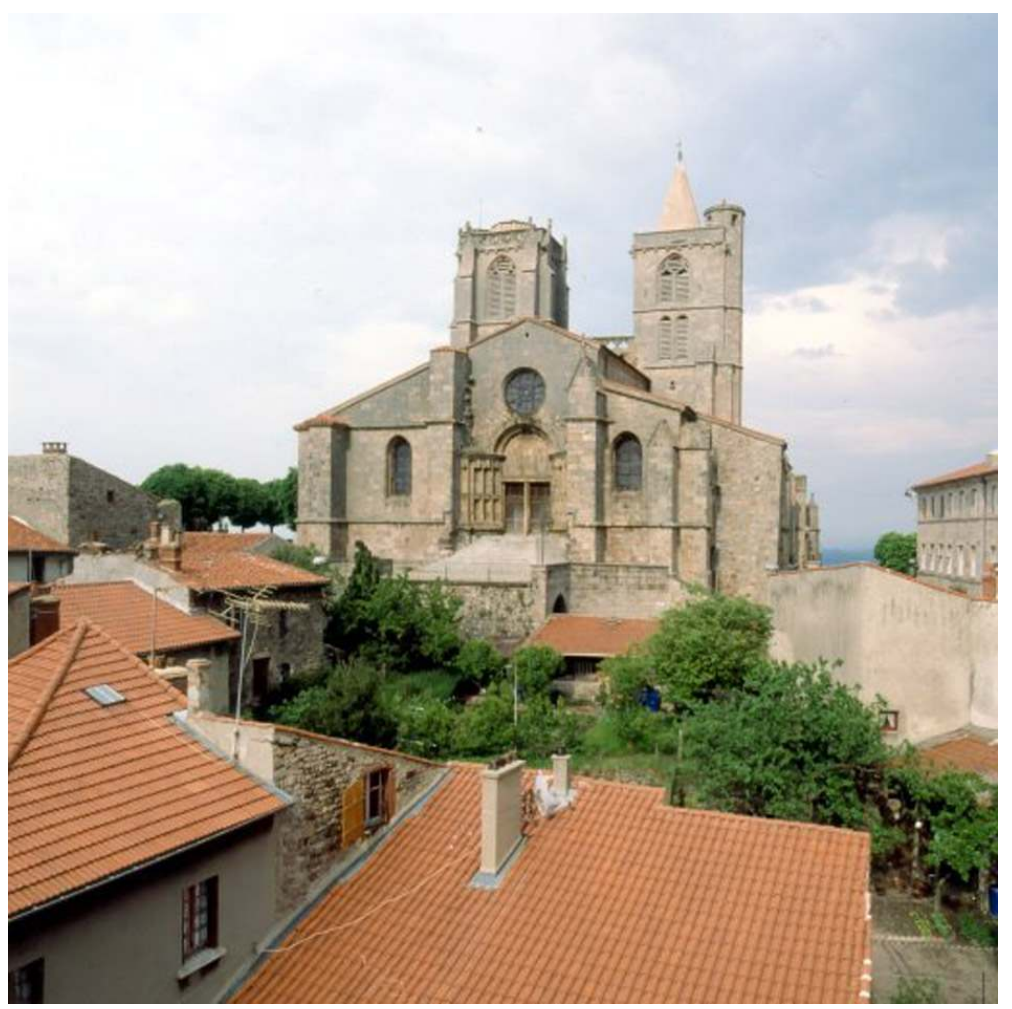

Vue générale de la façade ouest

Phot. Inv. E. Dessert. ( I Inventaire général, ADAGP, 1998

Le prolongement de la nef de deux travées vers l'ouest a entraîné, à cause de la pente du terrain, l'édification d'un vaste perron enjambant une rue et la construction, depuis celleci, de deux degrés latéraux pour atteindre la nouvelle façade principale (fig. $\left.n^{\circ} 11\right)$. Cette extension pose deux problèmes, l'un de structure, l'autre de datation.

Pour comprendre le premier, examinons d'abord le plan. La première travée est plus longue que les autres. A l'intérieur de celle-ci, le collatéral nord est plus large que son pendant, des murs plus puissants et une colonne plus épaisse le cantonnent. Sur l'élévation extérieure, la partie droite de la façade, correspondant au collatéral sud, est parfaitement cohérente: les contreforts sont amortis par des chaperons, une corniche sculptée souligne le pignon. A gauche, au contraire, les contreforts sont couronnés par des toits provisoires. Le portail central est ruiné: les arrachements permettent de restituer, au-dessus des ébrasements, une voussure surmontée d'une terrasse. La partie du pignon couronnant ces deux travées est refaite avec une pierre d'une teinte légèrement différente de celle d'origine et une fenêtre en oculus entaille, dans sa partie 
basse, les arrachements de la voussure du portail (fig. $\left.n^{\circ} 12\right)$. Sur l'élévation latérale de la travée nord le caractère d'inachèvement est encore plus lisible: quelques assises audessus du cordon supérieur sont interrompues par la toiture. Vers l'est, la présence d'un pan coupé, tout aussi brutalement arrêté dans son élévation, révèle vraisemblablement une cage d'escalier en vis. Le mur des chapelles suivantes (6 et 9) est parfaitement homogène alors qu'à l'intérieur, la première présente une voûte informe en contraste avec le beau couvrement d'ogives en pierre de taille de la seconde. Elles ont un accès unique par la troisième travée du collatéral.

Figure 12

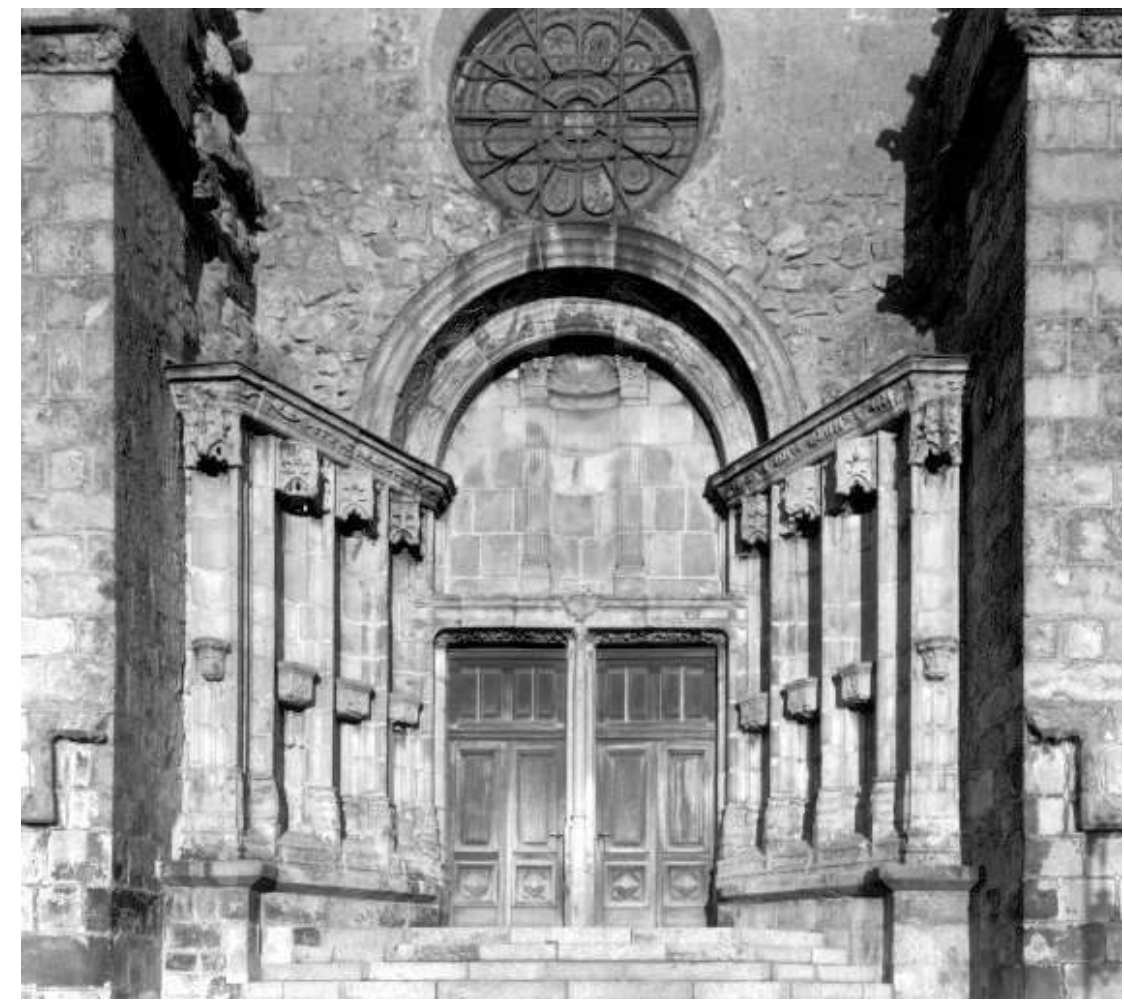

Portail ouest

Phot. Inv. E. Dessert. @ Inventaire général, ADAGP, 1998

De toutes ces observations il découle qu'un étage devait s'élever au-dessus de la première travée du collatéral nord ${ }^{24}$ et que les chapelles 6 et 9 n'en formaient primitivement qu'une, à deux travées. L'étage prévu est-il resté inachevé ? Les vilains arrachements de la voussure du portail et la voûte informe de la chapelle 9 témoignent indubitablement d'un accident et du rafistolage qui a suivi.

Quand cet accident a-t-il pu avoir lieu ? On pense naturellement aux guerres de Religion. L'église est mise à sac en 1562, à deux reprises, par le baron des Adrets. Les dégâts causés sont rapportés en 1622 par plusieurs témoins devant le châtelain de la ville : il n'est question que d'« église ravagée ", de mobilier pillé et de documents d'archives brûlés ${ }^{25}$. Les témoins, soixante ans après les faits, ont-ils omis de signaler une action aussi spectaculaire que la destruction d'une partie de l'église? C'est vraisemblable, car la finalité du document de 1622 se limite à la restitution des titres disparus. 


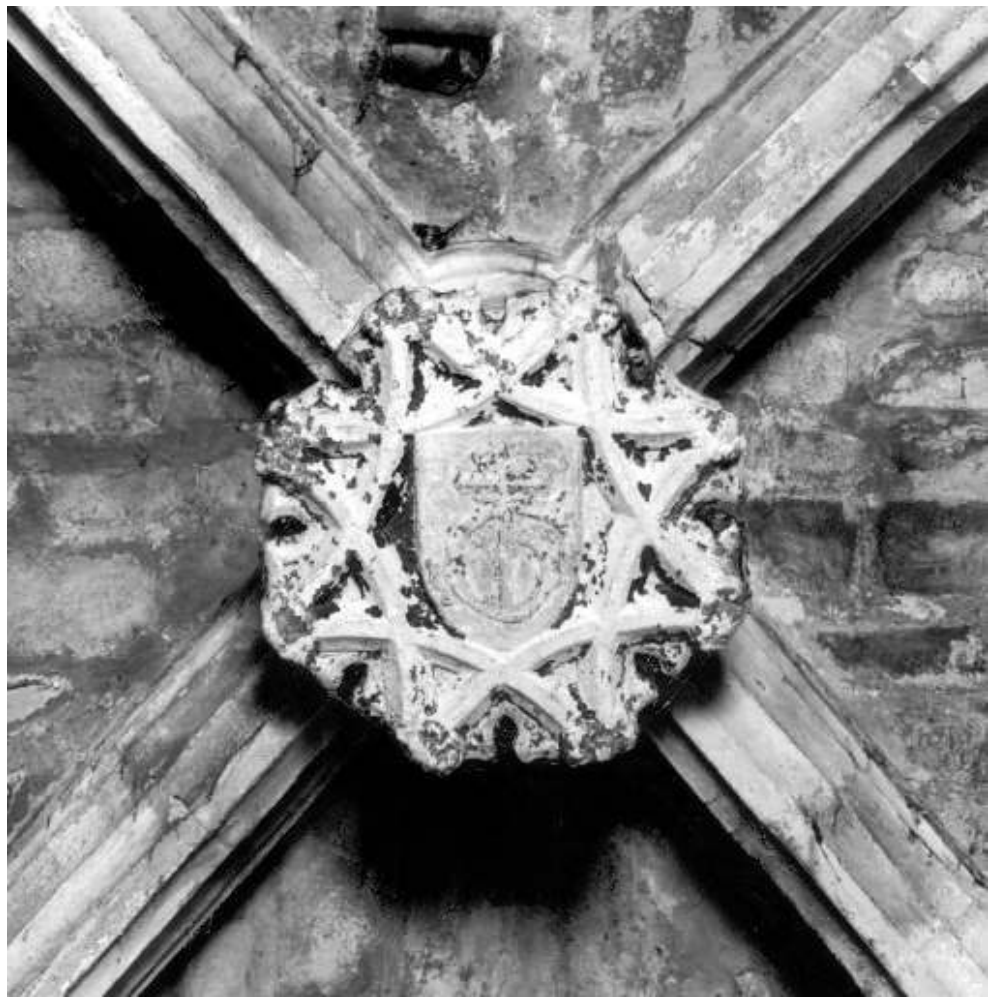

Nef, collatéral nord, clef de voûte de la deuxième travée

Phot. Inv. E. Dessert, J.-M. Refflé. (c) Inventaire général, ADAGP, 1998

Le deuxième problème à résoudre concernant le prolongement de l'église vers l'ouest est la date des travaux. Le portail ouest présente des ébrasements gothiques puis, à partir de l'imposte, un décor entièrement Renaissance. De là l'hypothèse a été émise que la partie basse serait un remploi du portail de la façade primitive ${ }^{26}$. La présence de feuilles d'acanthe sur les socles destinés à porter les statues dans cette partie rend peu plausible cette interprétation. En revanche, remarquons qu'à l'intérieur les clefs de voûte du collatéral nord ont un décor gothique (fig. $\mathrm{n}^{\circ} 13$ ), alors que celles des deux autres vaisseaux de l'agrandissement présentent des motifs de cuir découpé, de couronne et de fruits, en somme une ornementation qui n'est pas antérieure au milieu du XVI ${ }^{\mathrm{e}}$ siècle (fig. $\left.n^{\circ} 14\right)$. Aussi, le chantier a-t'il dû être conduit en deux phases : la première, à la fin du XV $\mathrm{XV}^{\mathrm{e}}$ ou au début du XVI e siècle, correspond au gros-oeuvre, aux voûtes du collatéral nord et à la partie inférieure du portail ; la deuxième, au milieu du XVI ${ }^{\mathrm{e}}$ siècle, se manifeste par l'achèvement du portail et le voûtement des deux autres vaisseaux. 


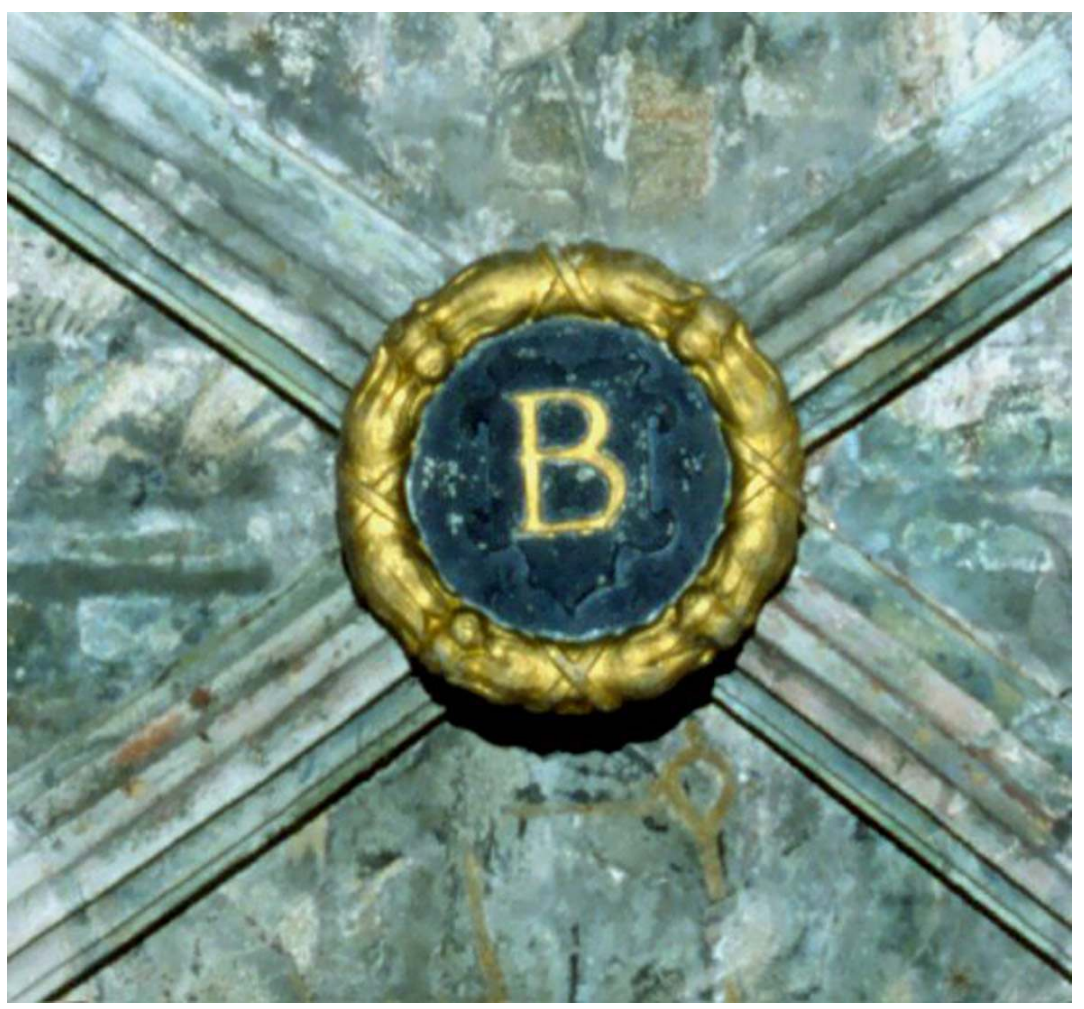

Nef, vaisseau central, clef de voûte de la deuxième travée

Phot. Inv. E. Dessert, J.-M. Refflé. (c) Inventaire général, ADAGP, 1998

L'analyse de ces quelques points sensibles permet ainsi de clarifier la chronologie de l'édifice. La reconnaissance des vestiges de la première église montre que celle-ci existe dès le XII ${ }^{e}$ siècle, antérieurement à la première mention de 1226, et qu'elle est ensuite remaniée au XIII ${ }^{e}$ siècle. Entre 1351 et 1361, son érection en paroisse et la création d'une communauté de prêtres sociétaires d'un caractère exceptionnel font vite ressentir la nécessité d'un lieu de culte plus vaste. La prospérité économique de la ville favorise le projet et les dons faits à l' "œuvre » en 1372 sont peut-être le témoin de la collecte de l'argent nécessaire à l'entreprise. Le chœur est déjà commencé en juin 1399 et le 1er mai de l'année suivante a lieu la cérémonie officielle de début des travaux, sans doute la pose de la première pierre. En 1418, le chantier est en voie d'achèvement du côté opposé puisqu'il est question des vantaux du portail ouest et qu'un clocher au moins paraît terminé. L'agrandissement de l'édifice de deux travées vers l'ouest est entrepris à la fin $\mathrm{du} \mathrm{XV}^{\mathrm{e}}$ siècle ou au début du XVI siècle, mais ne sera achevé, après un temps d'arrêt, qu'au milieu du XVI ${ }^{e}$ siècle ainsi qu'en témoignent les différences dans l'ornementation. L'examen de certaines particularités de la structure nous renseigne sur la présence de fonctions annexes au lieu de culte. Les légères différences constatées dans l'architecture des tours ne sont pas le fruit de la fantaisie mais correspondent à une utilisation variée : celle du sud est consacrée aux cloches, celle du nord, voûtée sur plusieurs niveaux, abrite une pièce à l'usage de la communauté, un logement sans doute pour le sonneur et un beffroi pour recevoir les plus grosses cloches. De plus, la circulation aisée sur l'ensemble des deux tours desservant des chemins de ronde couverts permettait d'assurer un rôle de guet efficace, essentiel dans le système de défense de la ville. Enfin, dans l'agrandissement réalisé vers l'ouest, du côté où s'étendait le quartier des prêtres, une 
salle assez vaste est construite à l'étage, de manière inhabituelle pour une église. Malheureusement, nous ignorons tout de sa destination.

\section{NOTES}

1. Fournial, Etienne. Les villes et l'économie d'échange en Forez aux XIII ${ }^{\mathrm{e}}$ et $\mathrm{XIV}^{\mathrm{e}}$ siècles. Paris : Les Presses du Palais Royal, 1967, p. 25-26, 134, 407, 414, 419, 424, 457, 484-485.

2. Costantini, Frédérique-Anne. Les comptes de La Chaise-Dieu. L'apport des sources écrites à l'étude de la construction d'un édifice. Les Dossiers de l'Archéologie, n²19, Les bâtisseurs du Moyen Age, 1996-1997, décembre-janvier, p. 92-97.

3. Dufour, J.-E. Dictionnaire topographique du Forez et des paroisses du Lyonnais et du Beaujolais formant le département de la Loire. Mâcon : impr. Protat frères, 1946, p. 852 ; [Condamin, James, Langlois, François]. Histoire de Saint-Bonnet-le-Château. Paris: A. Picard (...), 1885-1887, t. 1, p. 111-112.

4. Ibid., t. 1, p. 152-153.

5. Ibid., t. 1, p. 117-126, 147, 241.

6. Ibid., t. 1, p. 130-139. A.C. Saint-Bonnet-le-Château, L 1005.

7. [Condamin, James, Langlois, François], t. 1, p. 126, 130-139 ; t. 2, p. 79.

8. Langeon, Claude. La vie intellectuelle à Saint-Bonnet-le-Château au XVI ${ }^{\mathrm{e}}$ siècle. In Etudes foréziennes. III. Le passé des villes du Forez. Saint-Etienne: Centre d'études foréziennes, 1970, vol. 1, p. 177-185.

9. Selon la méthode préconisée par Eliane Vergnole lors de la première session du séminaire " La monographie d'architecture ", les 26 et 27 avril 2000 (séminaire sous la direction de Jean-Marie Pérouse de Montclos et organisé par la Direction de l'Architecture et du Patrimoine).

10. France. Inventaire général des monuments et des richesses artistiques de la France. Service régional de Rhône-Alpes. Canton de Saint-Bonnet-le-Château. Entre Forez et Velay. Réd. Bernard Ducouret, Thierry Monnet; photogr. Eric Dessert, Alain Franchella, Jean-Marie Refflé ; dess. Paul Cherblanc. Lyon : A.D.I.R.A., 1998 (Images du Patrimoine, $n^{\circ}$ 182), p. 45-47.

11. [Condamin, James, Langlois, François], t. 1, p. 228.

12. A.D. Loire : B 1870 .

13. Testament d'André Gurmeti, AD Loire, B $1870, \mathrm{f}^{\circ} \mathrm{LII} \mathrm{v}^{\circ}$. Condamin et Langlois citent à la même date un autre don à l'oeuvre par Pierre Patratz (t. 1, p. 151).

14. Fournial, p. 443.

15. A.D. Loire : B 1884, fol. XLVI.

16. [Condamin, James, Langlois, François], t. 1, p. 373.

17. Ibid., t. 1, p. 373-374 ; t. 2, p. 30, 234-235, 425-426.

18. Cf. supra.

19. Une nouvelle enceinte est entreprise en 1368 et des réparations ont lieu au château en 1400-1401 (Fournial, p. 443-445 ; Gonon, Marguerite. Extraits des comptes de la prévôté de Saint-Bonnet-le-Château rendus par Jean Syméon (1399-1404). In Documents linguistiques de la France (série franco-provençale) - XVIII. Documents linguistiques du Forez (1260-1498). Paris : CNRS, 1974, p. 366-369).

20. La cathédrale et la collégiale Saint-Paul à Lyon possèdent des logements en hauteur pour le sonneur, bien qu'ils soient des aménagements tardifs dans leur état actuel. 
21. Dubost, M., Epina, abbé. Les flèches en pierre ou brique en Forez, antérieures au XIX siècle. Bull. Diana, 1935, t. 25, p. 111-125.

22. Boyer, Pierre. Livre de raison du docteur Boyer, médecin à Saint-Bonnet-le-Château. Années 1620 à 1634. Publié par Louis Charréreau. Saint-Bonnet-le-Château: Prades-Freydier, [s.d.], p. 23.

23. [Condamin, James, Langlois, François], t. 2, p. 169-170.

24. Hypothèse déjà formulée par Carcel, Anne, Grange-Chavanis, Jean-François. Saint-Bonnet-leChâteau. Ancienne collégiale. Projet de restauration intérieure. Projet de restauration du parvis et du perron. Lyon: Conservation régionale des Monuments historiques, 1994. Etude préalable multigr., p. 25-26.

25. [Condamin, James, Langlois, François], t. 2, p. 103-106.

26. Carcel, Anne, Grange-Chavanis, Jean-François, p. 26.

INDEX

Mots-clés : inventaire général, en ligne, journal, revue électronique, revue numérique, périodique, patrimoine, histoire de l'art, France, Rhône-Alpes, architecture religieuse Keywords : on line, electronic journal, ejournal, heritage, history of art, France, architecture, collegiate churches, Rhône-Alpes

\section{AUTEUR}

\section{BERNARD DUCOURET}

Conservateur du patrimoine, Service régional de l'Inventaire DRAC Rhône-Alpes, Le Grenier d'Abondance 6, quai Saint-Vincent 69283 Lyon Cédex 01, 04.72.00.43.73.

bernard.ducouret@culture.gouv.fr 